\title{
Modelling frame losses in a two-hop ATM network
}

\author{
Zsehong Tsai, Kuo Hsin Yen \\ Department of Electrical Engineering, National Taiwan University, Taipei, Taiwan, ROC
}

Received 4 July 1994; accepted 25 November 1994

\begin{abstract}
Under the assumption that even a single cell loss could lead to a frame loss, we obtain formulas for the frame loss probability in a two-hop ATM network using two discrete time queueing models: the single queue model and the tandem queue model. Our results are also found to be much more accurate than the independent cell loss estimation.
\end{abstract}

Keywords: Queueing models; Frame loss probability; ATM network

\section{Introduction}

In recent years, significant advances in fast packet switching technology have made the development of local as well as wide area ATM networks possible. As in many legacy and other high-speed networks, the performance of ATM networks is essential for providing satisfactory services to users. Due to the slotted nature of ATM switching operations, many discrete time queueing models have been proposed to characterize the output queue behaviour in ATM switches and multiplexers. Most of these analytical models are used to investigate performance measures such as cell loss rate, mean cell delay and cell delay variation. The latter are often called the Quality of Service (QoS) performance parameters of the ATM networks. These results are used for other purposes, including call admission control or other preventive congestion control.

When higher layer QoS parameters are required, the above-mentioned ATM layer QoS performance parameters cannot usually be employed directly. For example, when an ATM backbone is used for LAN interconnection, which has been considered as one of the major applications of high-speed networks, the upper layer (such as the LLC layer) is more concerned with the loss probability of upper layer PDUs (frames), rather than the loss probability of an individual cell. Consequently, the translation of the ATM layer performance to the upper layer QoS parameters is suggested. Unfortunately, such translation is not a straightforward task, and any simplification such as the independent cell loss assumption could lead to an over-optimistic estimation, especially for bursty traffic sources [1]. Approaches which can obtain the desired QoS parameters, such as PDU loss probability for the upper layer, have thus become important issues. Nevertheless, in contrast to the large variety of existing discrete time queueing models for the analysis of cell loss rate, there are only a few papers dedicated to the estimation of upper layer PDU loss probability. Both discrete time and continuous time queueing models are considered by Cidon et al. [2]. However, under the discrete time model only the formulas for a single source are available. In addition, their models cannot be used when the cell stream is under a peak rate constraint. Both simulation results and upper bounds for the loss probability of upper layer PDUs are obtained by Lin and $\mathrm{Lu}$ [1]. The significant difference between the simulation results and the estimation based on independent cell loss assumption is also illustrated. But again, their results cannot be employed when there is a peak rate constraint. Both papers [1,2] consider the single stage queue model, which is good for modelling the output queue of a single ATM link, and assume that a single cell loss always lead to the loss of an upper layer PDU. The latter implies that the corresponding ATM Adaptation Layer can be of type $3 / 4$ [3].

Since an upper layer PDU can often be conveyed via a multiple-hop route in an ATM network with a mesh topology, any single stage queueing model should not be sufficient for characterizing the end-to-end frame loss statistics of the network. The study of frame loss 
probability in an appropriate discrete time tandem queue model is thus desired. In this paper, attention will be focused on the derivation of the frame loss probability when the ATM adaptation layer is of type $3 / 4$, and a two-hop ATM backbone is used for LAN interconnections or other bursty data communications. Our AAL type $3 / 4$ assumption is identical to the assumption that a PDU loss could result from a single cell loss. In addition, the frame traffic source is under a peak cell rate constraint, and cells of the same frame arrive in a burst. We employ two discrete time queueing models: a single queue model, used when the frame is conveyed across a single direct ATM link; and the tandem queue model, for the case when a two-hop route is used. The tandem queue model used here is similar to the model used in Ohba et al. [4] and D'Ambrosio and Melen [5]. However, our tandem queue model differs from those models $[4,5]$ in the sense that cells of the same frame are now specifically characterized as a tagged burst of cells. In both of our models, background traffic is chosen to be Bernoulli and ON_OFF cell streams, while the traffic load for the frame source is assumed insignificant. These two queueing models will be described in details in Sections 2 and 3. Exact iterative formulas for the frame loss probability of the single queue model are derived in Section 2, and the formulas for that of the tandem queue case are presented in Section 3. In Section 4 , we illustrate the numerical results based on the iterations and compare them with simulations. The impact of the peak rate constraint on the frame loss probability is also investigated.

\section{Single queue model}

In this section we investigate the frame loss probability at the output queue dedicated to a single link of an ATM switch. We assume that time is slotted for both input and output channels, and the slot size is exactly equal to a cell transmission time. The corresponding model is a discrete time single server queue with buffer size $M$, and it can be characterized by the following recurrence equation:

$Q_{m}=\min \left(Q_{m-1}+A_{m-1}, M\right)-I\left(Q_{m-1} \neq 0\right)$

where $I(\cdot)$ is the indicator function, $I(A)=1$ if $A$ is true; $I(A)=0$, otherwise; $Q_{m}$ is the number of buffered cells at the beginning of slot $m ; A_{m}$ is the number of arriving cells in slot $m$. In other words, the cells that arrive at the beginning of the slot have to wait for at least one slot, even when the server is idle.

We assume that the majority of the traffic load for the queue is contributed by its background traffic, which is assumed to be the superposition of cell streams from $l_{1}$ Bernoulli sources and $l_{2}$ ON_OFF sources, where $l_{1}$ and $l_{2}$ are arbitrary non-negative integers. Each Bernoulli source is assumed to generate a cell with probability $p$ for each slot. Each ON_OFF source is characterized as follows. It is a discrete time process alternating between two states: ON and OFF. When the process is at state OFF in the current slot, the probability that it will be at state $\mathrm{ON}$ in the next slot is given by $\omega_{1}$. When the process is in state $\mathrm{ON}$ at the current slot, the probability that it will be at state OFF in the next slot is given by $\omega_{2}$. A cell is generated with probability 1 when the process is in state ON, while no cell is generated if the state is OFF.

Under the above traffic conditions, we derive the loss probability for an $n$-cell framc, called the tagged frame, generated from a target source whose total traffic load is insignificant when compared with the background traffic. In addition, the target source is assumed to be subject to the peak rate constraint, such that the time interval between any two cells of the same frame is $S$ slots, $S \geqslant 1$ and $S$ is an integer. We also assume that a frame loss event occurs when one or more cells of this frame are discarded due to ATM switch buffer overflow. In calculating the loss probability, we assumed that the discarded cells are chosen randomly, with equal probability, from among those cells arriving in the same slot.

When the target source is excluded, the above queueing model is similar to the models considered in Ohba et al. [4] and Hou and Wong [6]. By defining the system state to be $(i, k)$, where $i$ is the number of buffered cells at the beginning of the slot and $k$ is the number of ON_OFF sources in the ON state, the system behaviour can be characterized as a discrete time Markov chain. Using the standard approach, we could denote the steady state probability for a system staying at state $(i, k)$ as $\pi_{i, k}$, and obtain the following balance equations:

$$
\begin{aligned}
\pi_{0, j}= & \pi_{0,0} B_{l_{1}}(0) \Gamma_{0, j}+\pi_{1,0} B_{l_{1}}(0) \Gamma_{0 j} \\
\pi_{i, j}= & \sum_{k=0}^{i} \sum_{l=0}^{k} \pi_{i+1-k, l} B_{l_{1}}(k-l) \Gamma_{l j} \\
& +\sum_{l=0}^{l_{2}} \pi_{0, l} B_{l_{1}}(i-l) \Gamma_{l j} \quad 1 \leqslant i \leqslant M-2, \\
\pi_{M, j}= & \sum_{k=M-l}^{\infty} \sum_{l=0}^{l_{2}} \pi_{0, l} \Gamma_{l j} B_{l_{1}}(k)
\end{aligned}
$$

where $B_{l_{1}}(k)$ is the probability of $k$ cells arriving from $l_{1}$ Bernoulli sources in a slot, $\Gamma_{k_{1} k_{2}}$ is the probability that $k_{2}$ sources will be at the ON state in the next slot, provided that $k_{1}$ ON_OFF sources are at the ON state in the current slot. $\Gamma_{k_{1} k_{2}}$ can be shown to be:

$$
\begin{aligned}
\Gamma_{k_{1} k_{2}}= & \sum_{i=0}^{k_{1}}\left(\begin{array}{c}
k_{1} \\
i
\end{array}\right) w_{2}^{k_{1}-i}\left(1-\omega_{2}\right)^{i}\left(\begin{array}{c}
l_{2}-k_{1} \\
k_{2}-i
\end{array}\right) \\
& \times\left(1-w_{1}\right)^{l_{2}-k_{1}-\left(k_{2}-i\right)} w_{1}^{k_{2}-i} \\
& \quad 0 \leqslant i \leqslant k_{2}, \quad 0 \leqslant k_{1}-i \leqslant l_{2}-k_{2}
\end{aligned}
$$


and:

$B_{l_{1}}(k)=\left(\begin{array}{c}l_{1} \\ k\end{array}\right) p^{k}(1-p)^{l_{1}-k} \quad$ for $\quad k=0,1, \ldots, l_{1}$

For the system of equations in (2), using the iterative method presented in [6], we find that the solution for $\pi_{i, k}$ can be obtained efficiently.

We are now ready to derive the iterative formulas for the loss probability of an $n$-cell tagged frame arriving at this queueing system in steady state. To make the notation easier to follow, we derive $L(n)$, the zero cell loss probability of an $n$-cell tagged frame, instead of directly obtaining the frame loss probability. We then categorize the traffic conditions into two cases: the consecutive case $(S=1)$ and the fixed-interval case $(S>1)$.

\subsection{Consecutive case}

We first consider the case that $S=1$, i.e. the cells of the tagged frame arrive at the queue with a rate equal to full bandwidth of the input channel. Obviously, we can obtain $L(n)$ by first calculating the conditional zero cell loss probability of the tagged frame given the system state observed by the first cell of this frame, and then derive the average zero cell loss probability $L(n)$. Thus, one could write:

$L(n)=\sum_{i=0}^{M} \sum_{k=0}^{l_{2}} \pi_{i, k} L_{i, k}(n)$

where $L_{i, k}(n)$ is the probability that there is zero cell loss for an $n$-cell frame, given that the system state when the first cell of the frame arrives is $(i, k)$. In this equation, we have employed the assumption that the target source traffic is insignificant compared to the background traffic, so that the leading cell of the tagged frame observes the system state with the probability distribution satisfying Eq. (2).

The following iterative formulas can be then used to derive $L_{i, k}(n)$. For $n=1$ :

$$
\text { if } \begin{aligned}
& 0 \leqslant i_{1} \leqslant M-l_{1}- l_{2}-1 \\
& L_{i_{1}, k_{1}}(1)=\left\{\begin{array}{l}
1, \\
\sum_{b=0}^{l_{1}} \operatorname{Pr}\left\{\tilde{A}<M-i_{1} \mid\right. \\
\left.A=k_{1}+b+1\right\} B_{l_{1}}(b)
\end{array}\right.
\end{aligned}
$$

otherwise where $A$ is the total number of arriving cells in a slot, $\tilde{A}$ is the number of cells from the background traffic sources that arrive at the queue within the same slot but reside in the buffer ahead of the cell belonging to the tagged frame, and:

$\operatorname{Pr}\{\tilde{A}<y \mid A=x\}= \begin{cases}\frac{y}{x}, & \text { if } y \leqslant x \\ 1, & \text { if } y>x\end{cases}$

Eqs. (6), (7) imply that arriving cells are randomly discarded when overflow occurs. For $n \geqslant 2$, one can use:

$$
\begin{aligned}
L_{i_{1}, k_{1}}(n)= & \sum_{k_{2}=0}^{l_{2}} \sum_{b=0}^{l_{1}} \operatorname{Pr}\left\{\tilde{A}<M-i_{1} \mid A=k_{1}+b+1\right\} \\
& \times L_{i_{2}, k_{2}}(n-1) B_{l_{1}}(b) \Gamma_{k_{1} k_{2}}
\end{aligned}
$$

where

$i_{2}=\min \left(i_{1}+k_{1}+b+1, M\right)-I\left(i_{1} \neq 0\right)$

\subsection{Fixed-interval case}

In the fixed-interval case, we assume that $S$, the interval between two cells of the tagged frame, is an integer larger than 1. To better characterize the system behaviour, we now divide the time axis into intervals of length $S$, with the beginning of the interval set to be the slot following the slot in which the cells of the tagged frame arrive, and index the $s$ th slots within the interval as slot- $s$, where $s=1,2, \ldots, S$. In other words, the slots in which cells of the tagged frame arrive are indexed as slot- $S$. We need to define the following new notation:

- $L_{i, k}^{s}(n): \operatorname{Pr}\{$ zero cell loss for an $n$-cell frame whose first cell will arrive at end of the current interval | system state at slot-s in the current interval is $(i, k)\}$ for $s=1,2, \ldots, S$.

- $L^{S}(n): \operatorname{Pr}\{$ zero cell loss for an $n$-cell frame whose first cell arrive at the current slot, which is indexed as slot- $S\}$.

The following iterative equations are then obtained in a fashion similar to Eqs. (5)-(8). For slots in which no cells of the tagged frame arrive, we have:

$$
\begin{aligned}
L_{i_{1}, k_{1}}^{s}(n)= & \sum_{k_{2}=0}^{l_{2}} \sum_{b=0}^{l_{1}} L_{i_{2}, k_{2}}^{s+1}(n) B_{l_{1}}(b) \Gamma_{k_{1} k_{2}} \\
& \text { for } \quad s=1,2, \ldots, S-1
\end{aligned}
$$

where

$i_{2}=\min \left(i_{1}+k_{1}+b, M\right)-I\left(i_{1} \neq 0\right)$

In Eq. (9), the initial condition described in Eq. (6) can be used for the case $n=1$, since $L_{i_{1}, k_{1}}^{s}(1)=L_{i_{1}, k_{1}}(1)$. Then, for slot- $S$, one can write:

$$
\begin{aligned}
L_{i_{1}, k_{1}}^{S}(n)= & \sum_{k_{2}=0}^{l_{2}} \sum_{b=0}^{l_{1}} \operatorname{Pr}\left\{\tilde{A}<M-i_{1} \mid A=k_{1}+b+1\right\} \\
& \times L_{i_{2}^{\prime}, k_{2}}^{1}(n-1) B_{l_{1}}(b) \Gamma_{k_{1} k_{2}}
\end{aligned}
$$

where

$$
i_{2}^{\prime}=\min \left(i_{1}+k_{1}+b+1, M\right)-I\left(i_{1} \neq 0\right)
$$


Finally, it is straightforward to obtain:

$$
L(n)=\sum_{i=0}^{M} \sum_{k=0}^{l_{2}} \pi_{i, k} L_{i, k}^{S}(n)
$$

\section{Tandem queue model}

In this section, we introduce an iterative numerical procedure to obtain the frame loss probability in a tandem queue model. The tandem queuc model consists of two stages. The stage 1 queueing model is identical to the single queue model described in Section 2, and the cells of the tagged frame can arrive consecutively or at a fixed interval. The output process for the tagged frame from the stage 1 queue is then considered as the input process of the tagged frame for the stage 2 queue, which is also similar to the model described in Section 2 except for the cell arriving pattern of the tagged frame. The background traffic conditions of stage 1 and stage 2 queues are assumed to be totally independent, i.e. they employ different background traffic sources and only those cells of the target source are conveyed across two queucs. In addition, the buffer size of the two stages can be different. The only additional analytical result required is the cell departure process of the tagged frame in stage 1 queue.

To proceed with the analysis for the stage 2 queue, we observe that the cell departure intervals of an $n$-cell tagged frame from the stage 1 queue is a sequence of random variables, $Z_{j}$, where $Z_{j}$ is interdeparture time between the $j$ th cell and $(j+1)$ th cell of the same tagged frame, $j=1,2, \ldots, n-1$. In this procedure, we also need to derive the stage 1 system state probability observed by the $j$ th cell is $(i, k)$, denoted as $\pi_{i, k}^{j}$, for $j=1,2, \ldots, n$. After the distributions for each $Z_{j}$ are obtained, and assuming independence among $Z_{j}$, the extension of the iterative formulas used for the single queue to incorporate the cell arrival patterns for stage 2 queue is then straightforward. In such calculations, we assume that the tagged frame is conveyed across the stage 1 queue without cell losses.

In the following, we first present the iterative formula for obtaining the probability distribution for $Z_{1}$. Here we index the slot at which the first cell of the tagged frame arrives as slot- 0 , and the $s$ th slot following slot- 0 and slot-s and the slot at which the second cell arrives as slot-S. Some new notation is defined as follows:

- $\tilde{Y}_{r}$ : the number of slots required to service those cells arriving during a period starting with slot- $r$ and ending at slot- $S$, and resides ahead of the second cell of the tagged frame, i.e. $\tilde{Y}_{r}=\sum_{s=r}^{S-1} Y_{s}+X_{S}$, where $Y_{s}$ is the number of slots required to service those cells arriving at slot-s, and $X_{S}$ is the number of slots required to service those cells arriving at slot- $S$ and residing ahead of the second cell of the tagged frame.

- $P_{\tilde{Y}_{1}, k_{1}, y, i^{\prime}, k^{\prime}}^{r}: \operatorname{Pr}\left\{\right.$ system state at slot- $S$ is $\left(i^{\prime}, k^{\prime}\right)$, $\tilde{Y}_{r}=y \mid$ system state at slot-r is $\left.\left(i_{1}, k_{1}\right)\right\}$, where $r=1,2, \ldots, S$.

- $P_{i_{1}, k_{1}, y, i^{\prime}, k^{\prime}}^{0}: \operatorname{Pr}\{$ system state at the slot in which the second cell of the tagged frame arrives is $\left(i^{\prime}, k^{\prime}\right)$, the number of slots between the departure instants of the first and second cells of the tagged frame is $y \mid$ system state at the slot when the first cell of the tagged frame arrives is $\left.\left(i_{1}, k_{1}\right)\right\}$.

The following iterative formulas are required to derive the interdeparture time for the first and second cells of the tagged frame. We start the derivation of the interdeparture time probability distribution conditioning on system state $(i, k)$ at the boundary slot, slot- $S$. Then we gradually extend the iterative formula until we can make use of the system state observed by the first cell of the frame. The boundary condition at slot- $S$ gives:

$P_{i, k, y, i^{\prime}, k^{\prime}}^{S}=\left\{\begin{array}{l}\sum_{b=0}^{l_{1}} T\left(i^{\prime}, k^{\prime}, b, y\right) B_{l_{1}}(b), \\ \text { if } i=i^{\prime}, k=k^{\prime}, i \neq 0 \\ \sum_{b=0}^{l_{1}} T\left(i^{\prime}, k^{\prime}, b, y-1\right) B_{l_{1}}(b), \\ \text { if } i=i^{\prime}, k=k^{\prime}, i-0 \\ 0\end{array}\right.$

otherwise where

$$
T\left(i^{\prime}, k^{\prime}, b, y\right)=\left\{\begin{array}{l}
\frac{1}{k^{\prime}+b+1}, \text { if } i^{\prime}+k^{\prime}+b+1<M \\
0 \leqslant y \leqslant k^{\prime}+b \\
\frac{1}{M-i^{\prime}}, \text { if } i^{\prime}+k^{\prime}+b+1 \geqslant M \\
0 \leqslant y \leqslant M-i^{\prime}-1
\end{array}\right.
$$

For the slots between the arriving instants of the first and second cell, we obtain:

$$
\begin{gathered}
P_{i_{1}, k_{1}, y, i^{\prime}, k^{\prime}}^{s}=\sum_{k_{2}} \sum_{b} \Gamma_{k_{1} k_{2}} B_{l_{1}}(b) P_{i_{2}, k_{2}, y-j-I\left\{i_{1}=0\right\}, i^{\prime}, k^{\prime}}^{s+1} \\
\quad \text { for } \quad s=1,2, \ldots, S-1
\end{gathered}
$$

where

$$
\begin{aligned}
i_{2} & =\min \left(i_{1}+k_{1}+b, M\right)-I\left(i_{1} \neq 0\right) \\
j & =\max \left(k_{1}+b, M-i_{1}\right)
\end{aligned}
$$


As for slot-0, we observe that:

$$
\begin{aligned}
P_{i_{1}, k_{1}, y, i^{\prime}, k^{\prime}}^{0}= & \sum_{j=0}^{k_{1} b b} \sum_{k_{2}} \sum_{h} \Gamma_{k_{1} k_{2}} B_{l_{1}}(b) P_{i_{2}, k_{2}, y-j, i^{\prime}, k^{\prime}}^{1} \\
& \times \frac{1}{k_{1}+b+1}
\end{aligned}
$$

where

$\dot{i}_{2}=\min \left(i_{1}+k_{1}+b+1, M\right)-I\left(i_{1} \neq 0\right)$

Next, we assume that the first cell of the tagged frame arrives at the queue when the system is in a steady state. This implies that $\pi_{i, k}^{1}=\pi_{i, k}$. The probability distribution for $Z_{1}$ is then given by:

$\operatorname{Pr}\left\{Z_{1}=x\right\}=\sum_{i=0}^{M} \sum_{k=0}^{i_{2}} \sum_{i^{\prime}=0}^{M} \sum_{k^{\prime}=0}^{l_{2}} \pi_{i, k} P_{i, k, x, i^{\prime}, k^{\prime}}^{0}$

In general, the system state observed by the $j$ th cell of the tagged frame is obtained as:

$\pi_{i^{\prime}, k^{\prime}}^{j+1}=\sum_{i=0}^{M} \sum_{k=0}^{l_{2}} \sum_{z} \pi_{i, k}^{j} P_{i, k, z, i^{\prime}, k^{\prime}}^{0} \quad j=1,2, \ldots, n-1$

while for the distribution $Z_{j}$, one can derive:

$$
\begin{gathered}
\operatorname{Pr}\left\{Z_{j}=x\right\}-\sum_{i=0}^{M} \sum_{k=0}^{l_{2}} \sum_{i^{\prime}=0}^{M} \sum_{k^{\prime}=0}^{l_{2}} \pi_{i, k}^{j} P_{i, k, x, i^{\prime}, k^{\prime}}^{0} \\
j=2,3, \ldots, n-1
\end{gathered}
$$

Using the above equations, the exact probability distributions of all $Z_{j}$ can be obtained in an iterative fashion. By assuming independence of $Z_{j}$, the procedure to iteratively calculate the zero cell loss probability of the $n$-cell tagged frame at the stage 2 queue is then a simple extension of the procedure described in Section 2.2.

\section{Numerical results}

First we consider the single queue model with a buffer size equal to 40 . The total background traffic load is set equal to 0.8 . Two traffic conditions are used: (T1) 5 Bernoulli sources, and (T2) 3 Bernoulli sources and 2 ON_OFF sources. For the ON_OFF sources, we set $\omega_{1}=\rho / 2$ and $\omega_{2}=(1-\rho) / 2$, where $\rho$ is traffic load of a single ON_OFF source, in all numerical examples. These two traffic patterns are used to study the impact
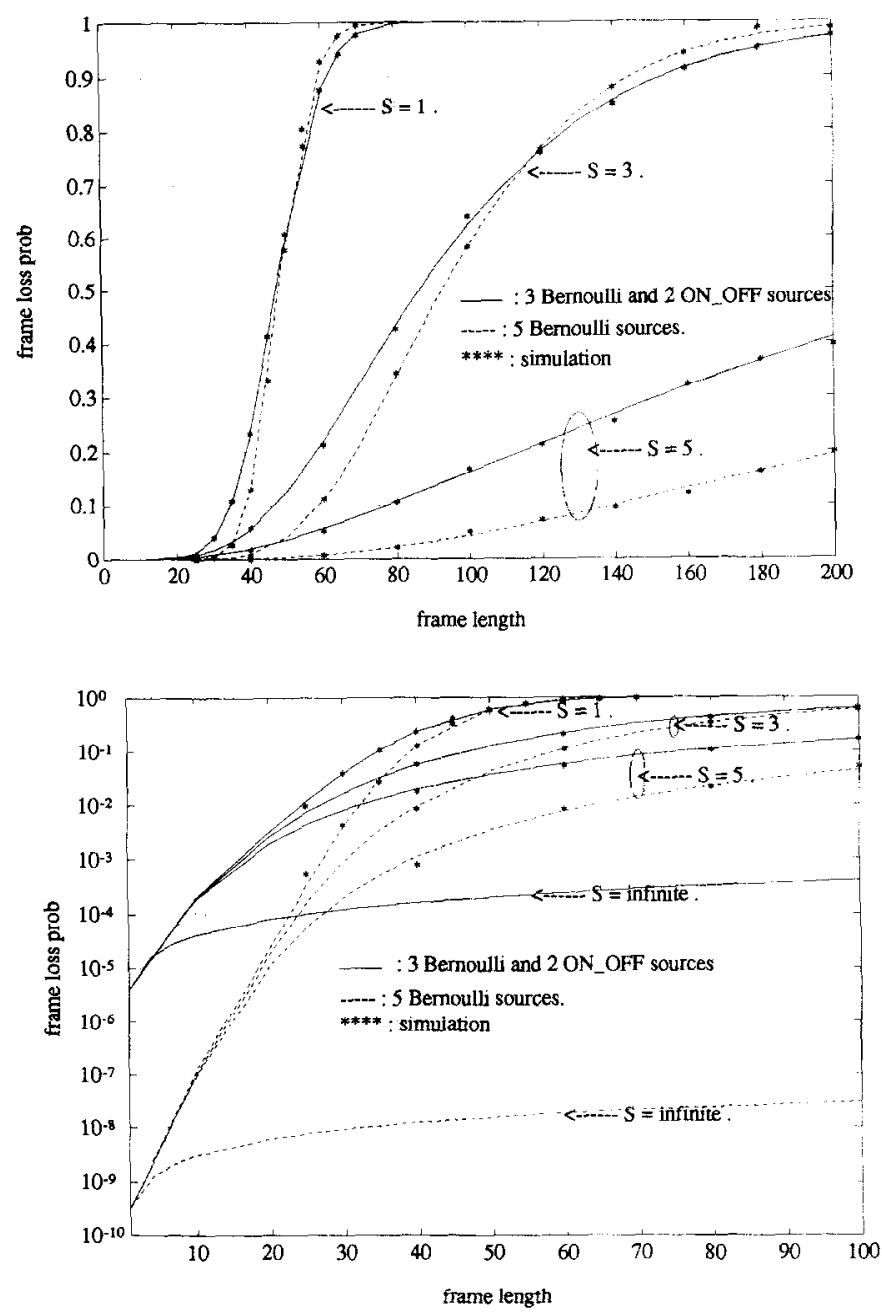

Fig. 1. (a) Frame loss probability vs. frame length. Corresponding parameters are buffer size $=40$, total load $=0.8$. (b) Frame loss probability vs. frame length. Corresponding parameters are buffer size $M=40$, total load $=0.8$.

of background cell stream patterns on the frame loss probability. In both conditions, all sources contribute the same amount of traffic load. The length of the tagged frame is assumed to vary from 20 to 200 cells, while the peak rate is set to be $1,1 / 3$ or $1 / 5$ of the input link bandwidth, i.e. $S=1,3$ and 5. In Figs. 1(a) and (b), using different scales for the frame loss probabilities, both analytical results and simulation statistics are presented. For the simulation, we have used a modified version of a Broadband ISDN simulation testbed developed in the EE Department of the National Taiwan University [7]. The analytical results are found to coincide with simulation statistics well, in all traffic conditions. In addition, as the peak cell rate decreases, or equivalently, when $S$ increases, the frame loss probability always drops drastically. This indicates that the use of peak rate control can easily lead to satisfactory performance results. One could note that the transmission time of the tagged frame is increased linearly to $S$, which 
might lead to a longer waiting time at the output queue of the frame source node. Nevertheless, we believe that the overall performance is still better as long as the required throughput is not close to the ATM link bandwidth, since fewer frame retransmissions are required, as indicated in the figures. In Fig. 1(b), the curve for infinitely large $S$ is corresponding to the frame loss probability obtained using the independent cell loss assumption, which is called the independent cell loss estimation in this paper. That is, the loss probability for an $n$-cell frame when $S \rightarrow \infty$ is simply estimated by $1-L(1)^{n}$, where $L(1)$ is given by Eq. (5). When the frame length is much larger than the queue buffer size $(=40)$, the ratio of the exact results and the independent cell loss estimation can be up to $10^{7}$. Obviously, the independent cell loss estimation is over-optimistic. Another interesting observation is related to the impact of background traffic patterns on system performance. We note that the existence of ON_OFF sources seems to have a
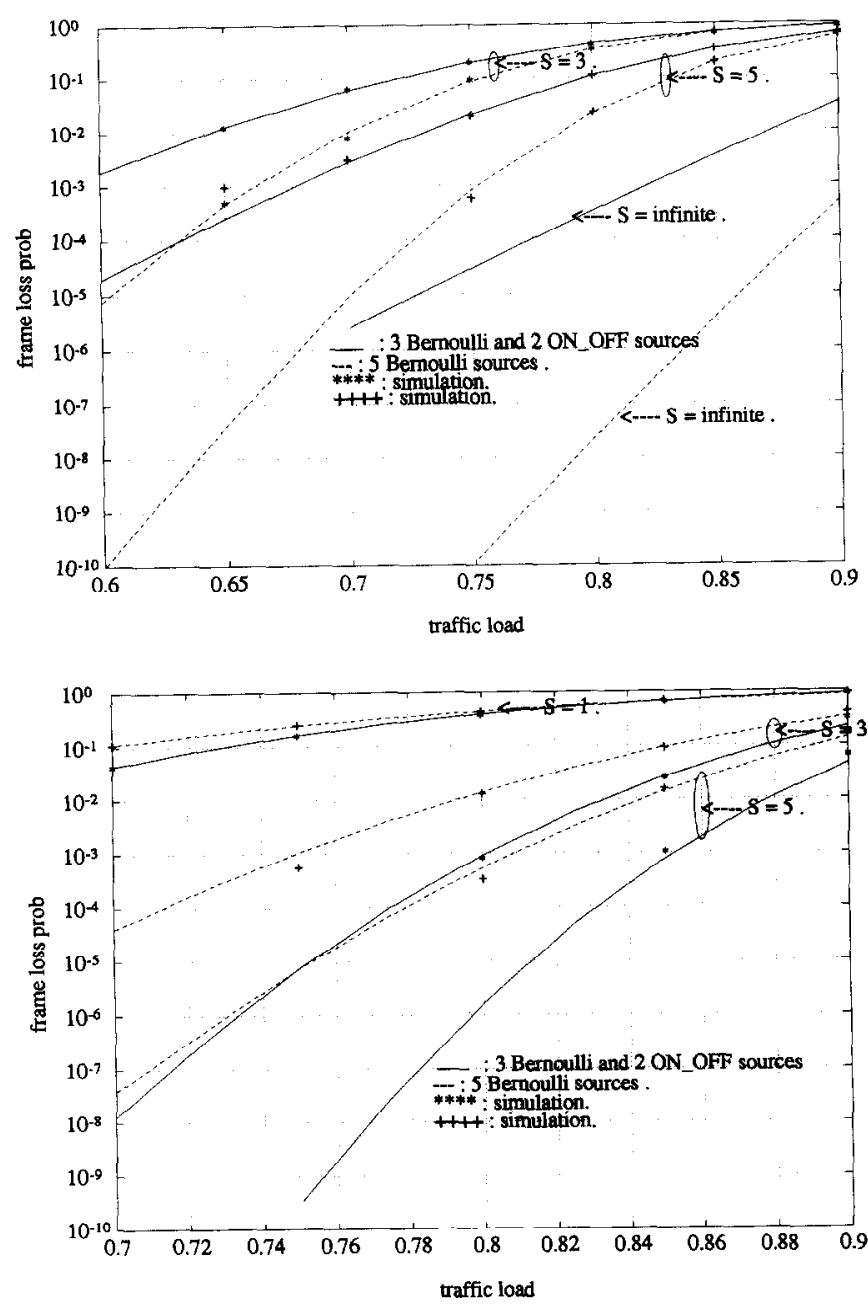

Fig. 2. (a) Frame loss probability vs. background traffic load. Corresponding parameters are buffer size $M=40$, frame length $=80$. (b) Frame loss probability vs. background traffic load. Corresponding parameters are: buffer size $M=100$, frame length $=120$.

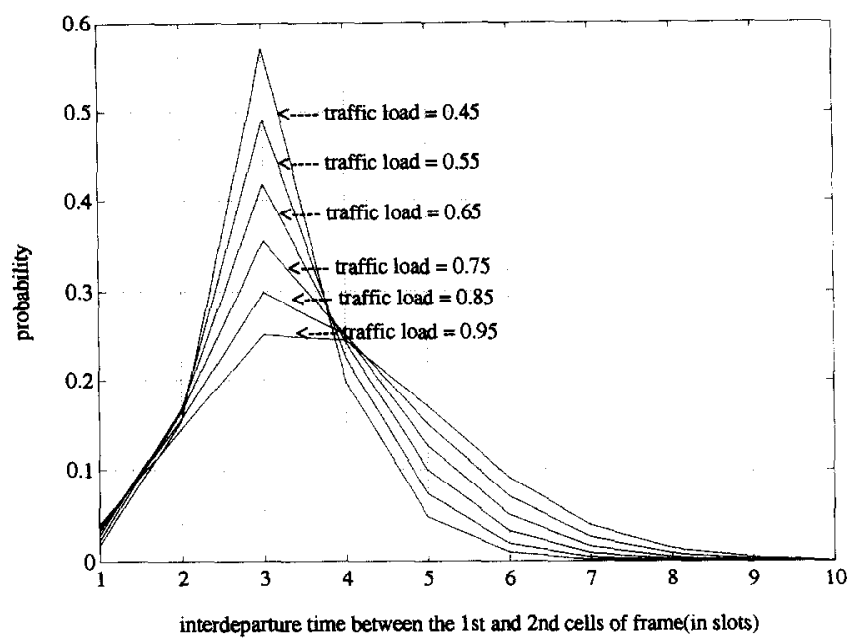

Fig. 3. Interdeparture time distribution for the first and second cells of the tagged frame. Corresponding parameters are buffer size $M=40$, total load varying from 0.45 to $0.95, S=3$ and five Bernoulli sources.

more serious effect on the frame loss probability when the frame loss probability is in an acceptable range (for example, under 0.1 ). This should be due to the fact that bursts generated by ON_OFF sources can easily cause buffer overflows, especially when its size is larger the queue buffer size, and this leads to frame losses. Only when the frame loss probability is exceedingly high, as shown in Fig. 1(a), could the use of Bernoulli sources lead to higher frame loss probability.

Next we plot the frame loss probability curves versus the traffic load in Figs. 2(a) and (b). In Fig. 2(a), the queue buffer size is still set equal to 40 but the frame length is 80 (cells). The same background traffic patterns as in Fig. 1 are used, while the total traffic load increases from 0.6 up to 0.9. In Fig. 2(b) we enlarge the buffer size to 100 and change the frame length to 120 (cells). Here the parameters $\omega_{1}$ and $\omega_{2}$ of the ON_OFF sources are adjusted according to the traffic load. Similar to what we observed in Fig. 1, the increase of $S$ can drastically decrease the frame loss probability under both background traffic conditions, and the simulation results still correspond well with the analytical results.

Before presenting the results obtained from the tandem queue model, we first make observations on the departure process of the single queue model using the formulas presented in Section 3. Typical interdeparture time distributions for the single queue model with $S=3$ and five identical Bernoulli background traffic sources are plotted in Fig. 3. The queue buffer size $M$ is 40 and the traffic load varies from 0.45 to 0.95 . As one might expect, when the background traffic load is close to 1 , the variance of the interdeparture time increases and the resulting departure process is very different from the arrival process, which is a switched periodic process. 


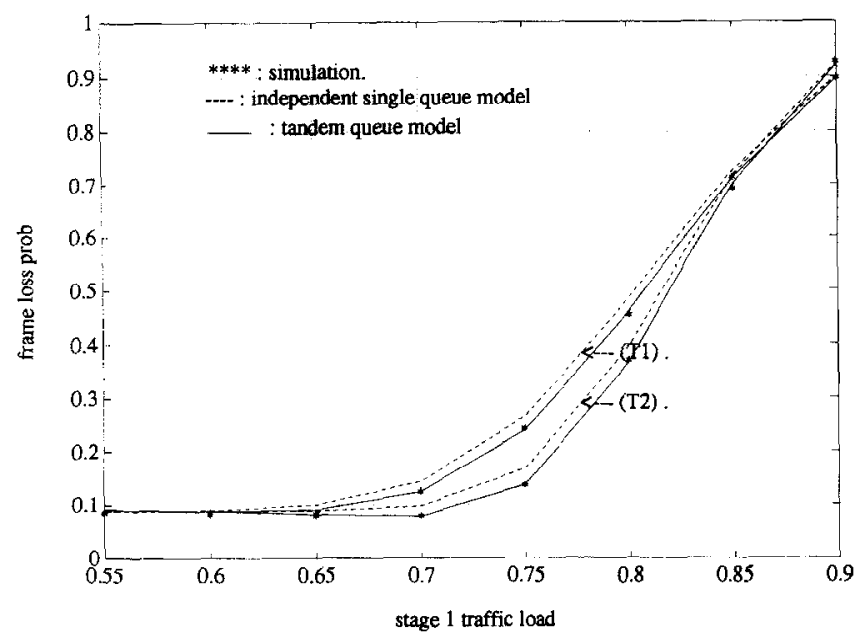

Fig. 4. Total frame loss probability vs. stage 1 traffic load. Corresponding parameters are: five identical Bernoulli sources whose total load is 0.75 in stage 2 . Stage 1 traffic sources are 3 Bernoulli and 2 ON_OFF sources (T1), or 5 Bernoulli sources (T2). Buffer size $M=40$ in both stages, frame length equal to 80 , cell interval $S=3$.

We now illustrate the accuracy of our method presented in Section 3 using the following tandem queue model. In stage 1 , there are 3 Bernoulli sources and 2 ON_OFF sources or 5 Bernoulli sources, with traffic load varying from 0.55 to 0.9 . Each of these five sources contributes $1 / 5$ of the stage 1 traffic load. In stage 2 , five identical Bernoulli sources are assumed, with total traffic load set equal to 0.75 . The queue buffer size $M$ is 40 for both stages, the frame length is assumed to be 80 and cell interval $S$ is 3 . The analytical results are plotted as solid curves in Fig. 4, and the simulation results are found to be very close to the results presented. We then compare our results with the following estimation method. Each stage of the tandem queue is modelled as an independent single server queuc, and the corresponding frame loss

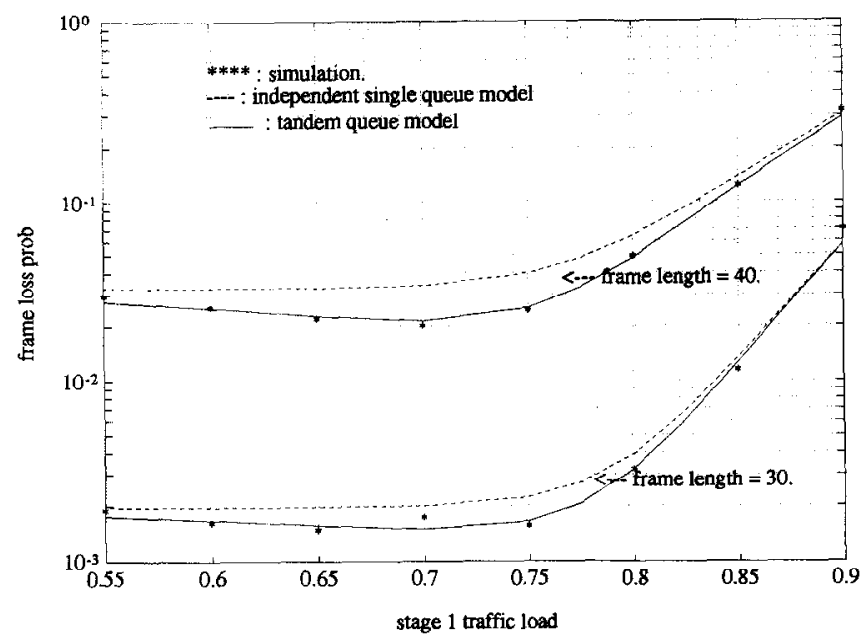

Fig. 5. Total frame loss probability vs. stage 1 traffic load. Corresponding parameters are: five identical Bernoulli sources whose total load is 0.8 in stage 2, five identical Bernoulli sources in stage 1, buffer size $M=40$ in both stages, frame length equal to 30,40 , cell interval $S=2$.

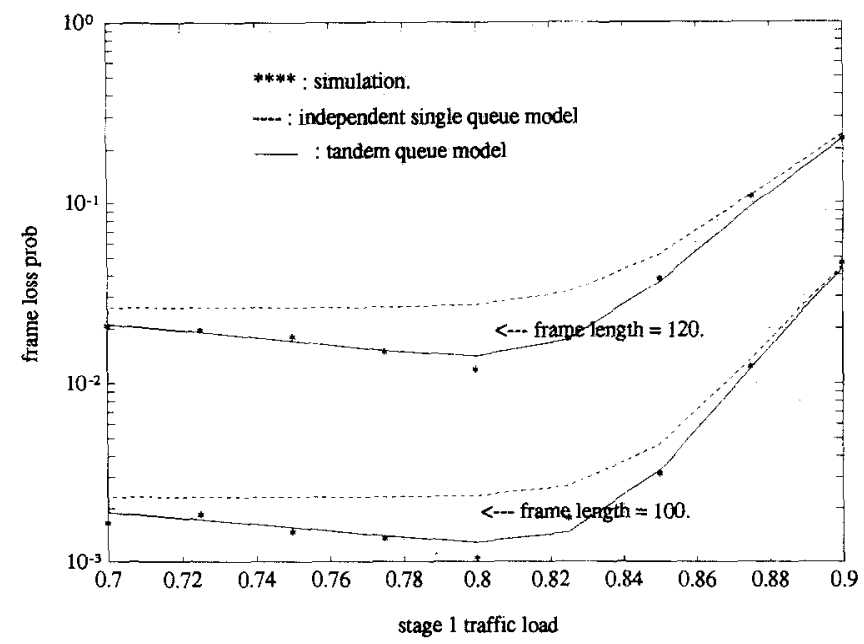

Fig. 6. Total frame loss probability vs. stage 1 traffic load. Corresponding parameters are: five identical Bernoulli sources whose total load is 0.85 in stage 2, five identical Bernoulli sources in stage 1, buffer size $M=100$ in both stages, frame length equal to 100,120 , cell interval $S=3$.

probability for each queue is calculated using the method described in Section 2, with the same tagged frame arrival pattern. The frame loss probability is then obtained assuming that the frame loss events at these two queues are independent. In the following, such approximations are said to be based on the independent single queue model and are plotted as dashed curves. The corresponding results are found to be less accurate, as one might expect. However, when the traffic load of stage 1 is exceedingly high or is light, the difference between these two models is less significant.

A different set of system parameters is used in Figs. 5 and 6 . In both stage 1 and 2, there are five identical Bernoulli sources. For Fig. 5, the stage 1 traffic load varies from 0.55 to 0.9 , while the stage 2 traffic load is set equal to 0.8 . The queue buffer size is 40 for both stages, the frame length is assumed to be 30 or 40 and cell interval $S$ is 2 . For Fig. 6, the stage 1 traffic load varies from 0.7 to 0.9 , while the stage 2 traffic load is set equal to 0.85 . The queue buffer size is 100 for both stages, the frame length is assumed to be 100 or 120 and cell interval $S$ is 3 . Here we observe similar phenomena as in Fig. 4, but the difference between the accuracy of the independent single queue model and the tandem queue model becomes more significant. In certain cases, the independent single queue model can lead to almost a $100 \%$ error in frame loss probability. Therefore, when the calculation time is allowed and the peak rate is close to ATM link bandwidth, we still suggest using the iterative method based on the tandem queue model.

In Fig. 7, the system parameters are similar to those used in Fig. 5, except that the frame length is set equal to 35 and $S$ is 1,3 and 5. Here, the difference between results obtained using the independent single queue 


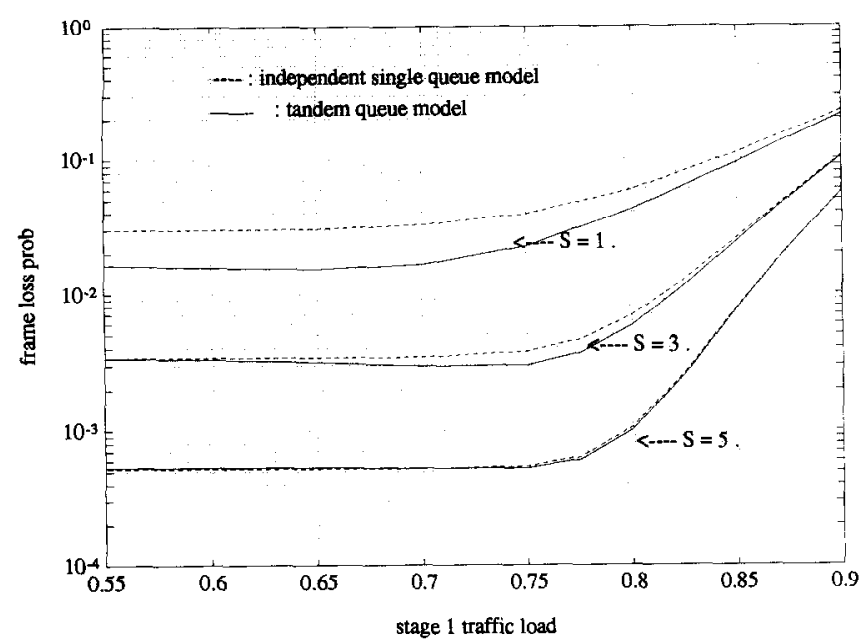

Fig. 7. Total frame loss probability vs. stage 1 traffic load. Corresponding parameters are: five identical Bernoulli sources whose total load is 0.8 in stage 2, five identical Bernoulli sources in stage 1 , buffer size $M=40$ in both stages, frame length equal to 35 , cell interval $S$ is 1,3 , and 5 .

model and those obtained using the tandem queue model is significant only for the set of curves corresponding to $S=1$ and 3 . For $S=5$, the difference is negligible. (For infinite $S$, we expect both models to yield results that are identical to the independent cell loss estimation.) Therefore, we believe that the independent single queue model is still useful when the peak rate for frame transmission is much smaller than the ATM link bandwidth.

\section{Conclusions}

In this paper, we present two queueing models for frame loss probability analysis in ATM networks and derive corresponding iterative formulas. Our formulas can provide exact frame loss probability for the single queue model and precise approximations for the tandem queue model. In both models, our approaches are found to yield far more accurate results when compared with estimation based on the independent cell loss assumption. Although only two types of traffic sources, the Bernoulli and ON_OFF sources, are considered, we believe that other types of sources can be incorporated when necessary. The results presented in this paper can thus be directly used in the end-to-end frame performance analysis when a two-hop ATM backbone is used for LAN interconnection or data communications.

Although our tandem queue model requires a more detailed calculation procedure, it always seems to lead to accurate results. We also provide an alternative approach to calculate the frame loss probability in a two-hop route, by assuming independence among the two stages in the tandem queue model. We found that when the peak rate used to transmit the tagged frame is much smaller than the ATM link bandwidth, or when the traffic load in one queue is small, then the independent single queue model can still yield accurate approximations. Otherwise, we still suggest the use of the detailed iterative approach, described in Section 3.

\section{Acknowledgements}

This work was supported in part by the National Science Council of Republic of China under Grants NSC 83-0416-E-002-012 and NSC 82-0416-E-002-249.

\section{References}

[1] F.Y.S. Lin and J.C. Lu, Estimating packet (L3_PDU) loss probabilities from cell (L2_PDU) loss probabilities for networks supporting the SMDS service, in 5th International Conference on Data Communication Systems and their Performance, 1993.

[2] I. Cidon, A Khamisy and M. Sidi, Analysis of packet loss processes in high-speed networks, IEEE Trans. Information Theory, 39 (1) (January 1993) 98-108.

[3] G.J. Armitage and K.M. Adams, Packet reassembly during cell loss, IEEE Network, (September 1993) 26-34.

[4] Y. Ohba, M. Murata and H. Miyahara, Analysis of interdeparture process for burst traffic in ATM networks, IEEE J. Select. Areas Commun., 9 (3) (April 1991) 468-476.

[5] M. D'Ambrosio and R. Melen, On the modification of the cell streams within an ATM network, Globecom '93 1993, pp. 1334-1340.

[6] T.-C. Hou and A.K. Wong, Queueing analysis for ATM switching of mixed continuous-bit-rate and bursty traffic, in Proc. INFOCOM'90, 1990.

[7] J.-F. Chang et al., Design, implementation, and applications of a B-ISDN simulation testbed, in Proc. 28th Annual Simulation Symposium, 1995.

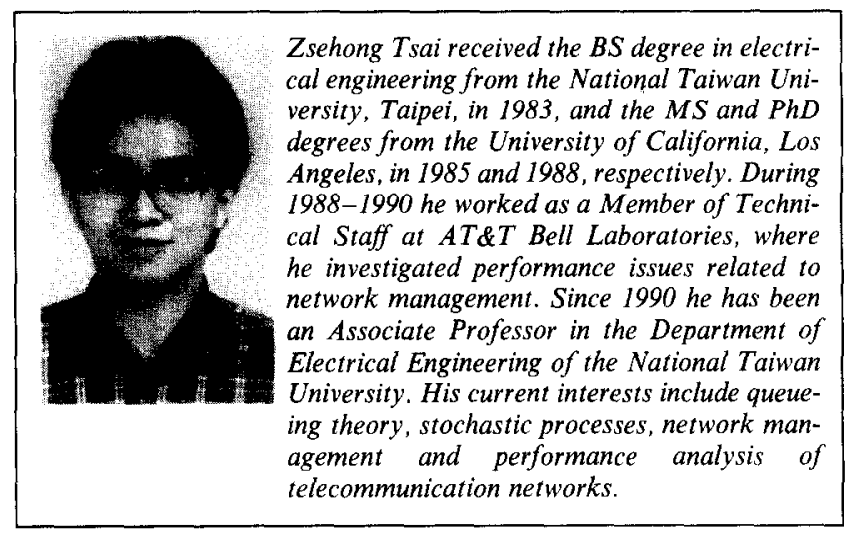

Kuo Hsin Yen received the BS degree in electrical engineering from the National Tsing Hua University, Hsinchu, in 1992, and the MS degree from National Taiwan University, Taipei, in 1994, respectively. His current interests include queueing theory and high speed networking. 